\title{
Cooperation in Intergroup, $N$-Person, and Two-Person Games of Chicken
}

\author{
GARY BORNSTEIN \\ Department of Psychology \\ Hebrew University of Jerusalem \\ DAVID BUDESCU \\ Department of Psychology \\ University of Illinois at Urbana-Champaign \\ SHMUEL ZAMIR \\ Department of Statistics \\ Hebrew University of Jerusalem
}

The authors introduce a new team game, the intergroup chicken game, to model intergroup conflicts involving bilateral threats (e.g., military conflicts, industrial disputes). The group that wins the game is the one that competes while the other group yields, and the benefits associated with winning (e.g., territory, higher wages) are public goods for the members of that group. However, a failure to yield on the part of both groups leads to an outcome (e.g., war, strike) that is disastrous to all the players. The authors report an experiment in which an intergroup chicken game with two players on each team was compared to a two-person chicken game and a (single-group) four-person chicken game. The games were played repeatedly, and each round was preceded by a signaling period. Results showed that subjects were more competitive (and, consequently, less efficient) in the intergroup chicken game than in either the two-person or the four-person chicken game.

$\mathbf{T}$

The two-person game of chicken derives its name from the following story. Two drivers race toward each other on a narrow road. Each driver has the choice to swerve and avoid a head-on collision or to continue on the collision course. Much of the interest in this game has stemmed from questions concerning intergroup and international relations rather than interpersonal relations. The two-person chicken game has been used to model a variety of intergroup conflicts involving bilateral threat, ranging from military confrontations between states to disputes between management and workers (Allison 1971; Brams 1975; Snidal 1991). These conflicts are analogous in the sense that a failure on the part of both sides to yield leads to an outcome, such as war or bankruptcy, that is disastrous to both.

AUTHORS' NOTE: This research was supported by grants from the Israel Science Foundation (94-97) and the Israel Foundation Trustees (94-96). We thank Yael Zick for her help in collecting the data. Correspondence concerning this article should be addressed to Gary Bornstein, Department of Psychology, Hebrew University, Jerusalem 91905, Israel; e-mail: MSGARY@pluto.mscc.huji.ac.il.

JOURNAL OF CONFLICT RESOLUTION, Vol. 41 No. 3, June 1997 384-406

(C) 1997 Sage Publications, Inc.

384 
The use of a two-person game to model conflict between groups presupposes that all group members have identical preferences over the set of possible outcomes and therefore that each group can be treated as a unitary player. The unitary player assumption, however, collapses when, as is often the case, the benefits associated with the outcome of the intergroup conflict (e.g., territory, higher wages) are public goods that, at least to some degree, are nonexcludable with respect to the members of a group involved in the competition. Thus, whereas as a collective all group members benefit from winning the competition and acquiring these goods, rational and selfish individuals who take a "free ride" (e.g., declining to fight, standing on a picket line) gain more (Taylor 1987).

The goal of this study is to examine the internal problem of collective action in intergroup conflicts of this type and its effects on conflict resolution. Following the pioneering work of Palfrey and Rosenthal (1983) on voting behavior, we model intergroup conflict as a team game. A team game entails a competition between two groups or teams labeled $A$ and $B$ with $n_{A}$ and $n_{B}$ members, respectively. Each member of team $A(B)$ receives an endowment of size $e$ and then must decide privately whether to contribute the endowment to his or her team's effort. Denote the number of contributors in teams $A$ and $B$ by $m_{A}$ and $m_{B}$, respectively $\left(0 \leq m_{A}<n_{A}\right.$ and $0 \leq m_{B}<$ $\left.n_{B}\right)$. If $m_{A}>m_{B}\left(m_{A}<m_{B}\right)$, then each member of team $A(B)$ receives a payoff of $r$ units. If $m_{A}=m_{B}$, then each player on both teams receives a payoff of $s$ units. Members of the losing team receive no reward, and contributions are not refunded.

A general definition of the intergroup chicken game is provided by the following pair of inequalities involving the payoff parameters: $r>s+e$ and $s<e$. The first expresses the fact that being on a winning team, even at the cost of contributing one's endowment, is strictly better than keeping one's endowment and not being on a winning team. The second inequality means that, unless a player ends up on a winning team, he or she would rather keep the endowment.

In the present study, the game is described as a competition between two teams of two members each. Each player receives an endowment of $e=2$ points (to be converted into money) and has to decide between keeping the endowment or "investing" it. A reward of $r=5$ points is given to each member of a team if the number of investors on his or her team exceeds that on the other team. Members of the losing team receive nothing. If there is an equal number of investors on both teams, then the players receive no bonus $(s=0)$. Regardless of the outcome of the game, players who do not invest their endowments keep them. Table 1 presents the payoff matrix for the intergroup chicken game.

We report an experiment in which the intergroup chicken game was contrasted with two-person and four-person chicken games. Our interest is in assessing the ability of the participants in the different games to achieve the cooperation necessary to the realization of their mutual interests. In the two-person chicken game, each player receives an endowment of 2 points and has to decide whether to keep the endowment or invest it. A reward of 5 points is provided to a player who invests although the other player does not. If both or neither of the players invest, then neither receives a bonus. A player who does not invest his or her endowment gets to keep it. Note that this is 
TABLE 1

Intergroup Chicken Game

Team A

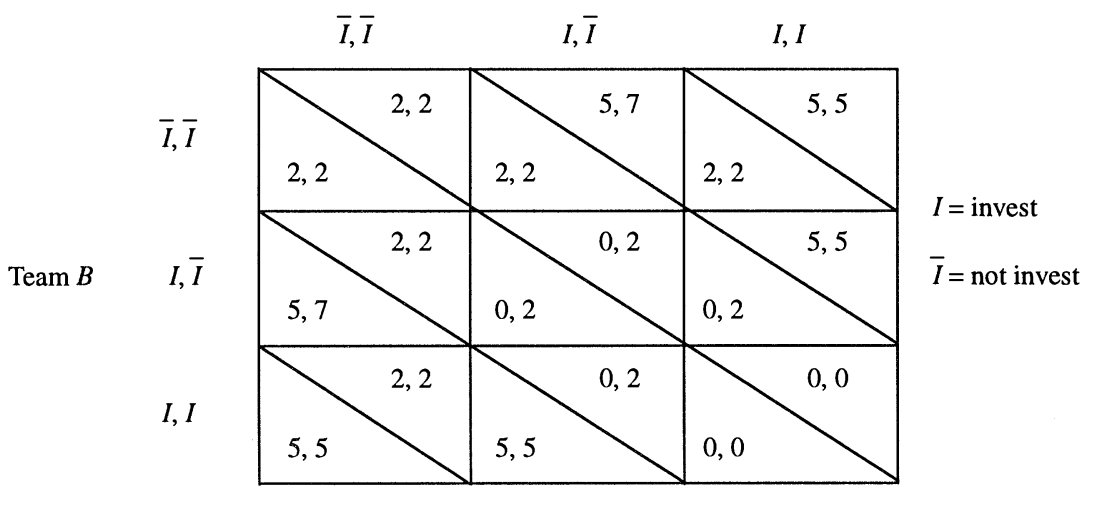

NOTE: The payoffs above the diagonals are for the members of team $A$, whereas those below the diagonals are for the members of team $B$. The first payoff is for the left member of the team, and the second is for the right member.

TABLE 2

Two-Person Chicken Game

Player $i$

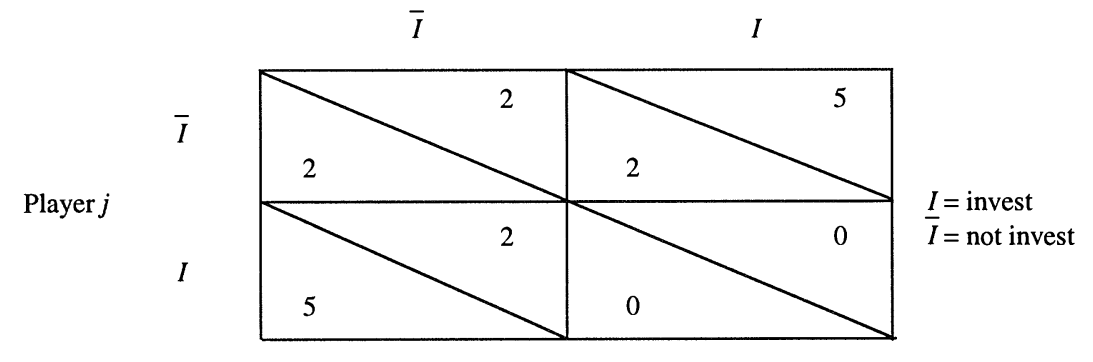

NOTE: The payoffs above the diagonals are for player $i$, whereas those below the diagonals are for player $j$.

identical to the previous team game if each team consists of a single player. The payoffs are presented in Table 2 . $^{1}$

1. Using the notations $x$ for the cooperative outcome (both players not investing), $w$ for the competitive outcome (both players investing), $y$ for the free-rider payoff (the payoff to a single investor), and $z$ for sucker payoff (the payoff to a single noninvestor), the traditional conditions defining a chicken game are $y>x>z>w$. In our case, the conditions satisfied are $y>x=z>w$. It can easily be verified that the game of chicken retains all of these strategic properties under these conditions as well. 
TABLE 3

Four-Person Chicken Game

\begin{tabular}{llllll}
\hline \hline $\mathrm{m}$ & 0 & 1 & 2 & 3 & 4 \\
\hline Invest & & 2.5 & 2.5 & 2.5 & 2.5 \\
Not invest & 2 & 4.5 & 4.5 & 4.5 & \\
\hline
\end{tabular}

NOTE: The payoff to player $i$ is a function of his or her decision and of the total number of investors $m$.

In the four-person chicken game, each player receives an endowment of 2 points and has to decide between keeping the money or investing it. A reward of 2.5 points is provided to each of the four players if at least one of them invests his or her endowment. If no one invests, then the players receive no reward. As in the other conditions, players who do not invest their endowments keep them. Table 3 presents the payoffs in this four-person chicken game.

The three variants of the chicken game are comparable in two important aspects. First, the Nash equilibria in pure strategies in all three games are such that only a single player invests (see Appendix for a complete specification of the games' Nash equilibria). Second, the pure-strategy equilibria are Pareto efficient; that is, these strategy combinations maximize the sum of payoffs to all the individuals involved. ${ }^{2}$ This property implies that, as a collective, all players have an interest in coordinating their actions on a pure-strategy equilibrium. However, because any player can assume the role of the single investor, each game has multiple equilibrium points. How do players choose among these alternative equilibria?

If the game is played only once and choices are made simultaneously and without communication, then the players have no way of choosing. Indeed, under such conditions, they can hardly be expected to settle on one of the pure-strategy equilibria. Instead, it can be argued, they might settle on a mixed-strategy equilibrium (e.g., Rasmusen 1989). As we prove in the Appendix, all three chicken games have a unique symmetric mixed-strategy equilibrium that does not distinguish among the players and can therefore serve as a focal point for solving the coordination problem presented by the game. ${ }^{3}$ However, the uncoordinated use of a mixed strategy has a major disadvantage: it leads to a Pareto-deficient outcome and is therefore wasteful in comparison to the pure-strategy equilibria.

One device that could prove useful for solving this coordination problem is "cheap talk" or signaling - costless communication that takes place outside the specification of the game. As suggested by Palfrey and Rosenthal (1991), cheap talk can be beneficial in settings where the potential gains for coordinated behavior are substantial. Indeed, a simulation by Ward (1990) demonstrated that joint gains are likely to be realized in a three-person game of chicken if the game is preceded by a pregame phase

2. An outcome is Pareto efficient (or Pareto optimal) if there is no other outcome with greater or equal payoffs to all players and a strictly higher payoff to at least one player.

3. The symmetric mixed-strategy equilibria of the intergroup, two-person, and four-person games of chicken, as described here, are for each player to invest with probabilities of $.42, .60$, and .07 , respectively (see Appendix). 
in which the players can signal their commitment to a certain course of action. Similar effects of nonbinding communication on cooperation were found in the experimental studies of the minimal contribution set paradigm - an $n$-person game of chicken (van de Kragt, Orbell, and Dawes 1983).

Another coordination device that could improve the prospects of cooperation is repetition of the game. If the players repeat the game time after time, then they eventually may settle on one of the Nash equilibria even in the absence of communication. Repetition is important for yet another reason: it enables players to achieve fair and equal payoffs in addition to Pareto efficiency. Individuals, as well as profitmaximizing firms (see Kahneman, Knetsch, and Thaler 1986), care about being treated fairly and are willing to resist unfair treatment by others even at some cost to themselves. An iterated setting in which current behavior depends on the earlier choices of other players enables them to do that. Ideally, an optimal and fair outcome can be achieved by alternating between the pure-strategy equilibria such that each player invests on every second round in the two-person game and on every fourth round in the intergroup and four-person games.

The mathematical formulations of the repeated interaction outcomes are given by the folk theorem, which states that the set of (subgame perfect) equilibrium payoffs in the infinitely repeated, undiscounted game is the set of individually rational payoffs in the convex-hull of the payoffs in the one-stage game (e.g., Rubinstein 1980).

Figure 1 illustrates the folk theorem for the two-person chicken game but not for the four-person games (due to lack of competence in drawing four-dimensional figures).

The one-stage payoffs are $(2,2),(5,2),(2,5)$, and $(0,0)$. When the convex-hull of these points is intersected with the individual rationality conditions $x_{i} \geq 2, i=1,2$ (each player can guarantee 2 by not investing), we obtain the shaded triangle in Figure 1 with vertices $(5,2),(2,5)$, and $(2,2)$. Each point in this triangle can be sustained as an equilibrium outcome of the repeated game. In particular, this is true for efficient outcomes, namely the points on the Pareto-optimal frontier-the line segment $[(5,2)$, $(2,5)]$. For example, the symmetric (i.e., "fair") efficient outcome $(3.5,3.5)$ is obtained by investing alternately, in turns, to obtain the string of payoffs $(5,2),(2,5),(5,2),(2$, 5 )... (with the threat never to invest again as soon as the other player deviates from this coordination pattern). Unlike the efficient $(3.5,3.5)$, which requires the coordination of the two players, the inferior payoff $(2,2)$ is achievable as an equilibrium payoff of the repeated game with no need for coordination. At each stage, each player invests with probability .6; that is, the mixed equilibrium of the one-stage game, $(.6, .6)$, is played repeatedly. Note that the payoff $(2,2)$ also can be achieved if both players never invest, but this is not an equilibrium behavior.

Table 4 lists, for each of the three games, all the symmetric equilibria in the repeated game that are based on equilibria of the one-stage game. An equilibrium of the one-stage game is given as a vector of probabilities (to invest) for each of the players. An asterisk added to such a vector indicates a strategy in the repeated game in which the cyclic permutations of the vector are played successively. Thus, $(1,0) *$ stands for the pattern $(1,0),(0,1),(1,0), \ldots$, and $(.42, .42, .42, .42)^{*}$ means to play repeatedly (and independently) the mixed strategy $(.42, .42, .42, .42)$. Finally $(.6, .6 ; 0,0) * *$ means 


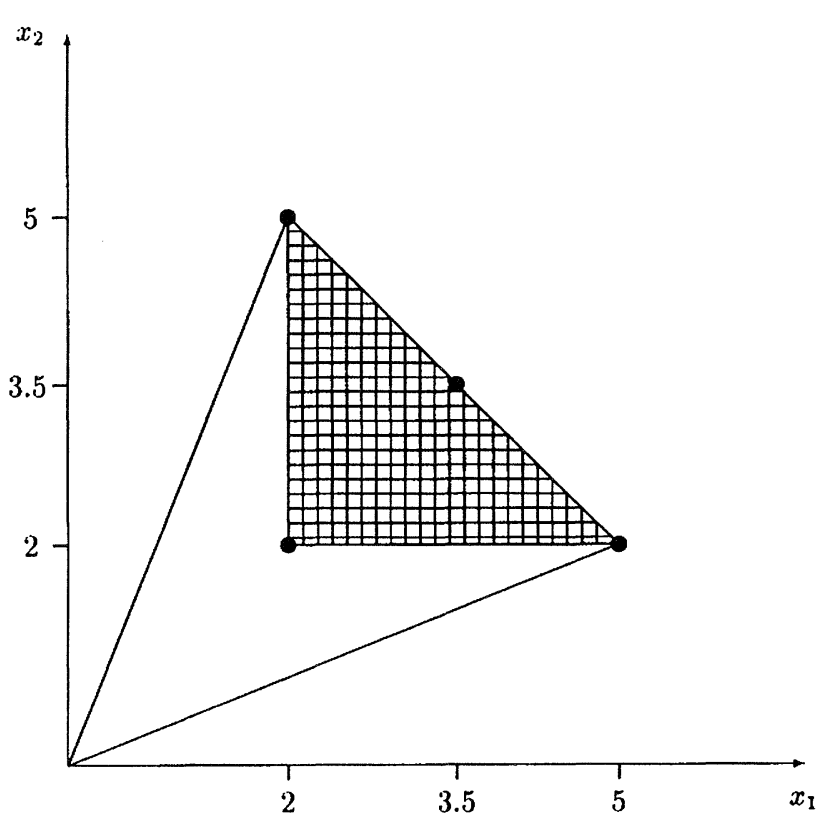

Figure 1: Equilibria in the Repeated Two-Person Chicken Game

that the two groups take turns in the role of investors; that is, $(.6, .6 ; 0,0),(0,0 ; .6$, $.6),(.6, .6 ; 0,0) \ldots$ Within each game, the solutions are listed according to their expected payoffs (from highest to lowest).

To each of the equilibria in Table 4, we indicated the expected payoff per player (per stage), the percentage of stages in which an individual invests (on average), and the level of coordination needed to execute the equilibrium. The important feature of Table 4 for our purposes is that, in all games, the efficient symmetric equilibrium requires the highest level of coordination (namely taking turns). On the other extreme, repeating the same, one-stage, mixed strategy requires no coordination whatsoever and yields the lowest expected payoffs.

In the present experiment, the games were, of course, finitely repeated. However, although the participants were not informed about the exact length of the games, they knew that the games involve a large number of repetitions. These conditions approximate, at least roughly, the conditions for the application of the folk theorem. Each repetition was preceded by a pregame period in which the players could signal their intentions to invest or not. Given the opportunity and the means of coordination, we compared the relative success of the participants in the different games in achieving an optimal and fair outcome. 
TABLE 4

Symmetric Equilibria in the Repeated Games

Based on Equilibria in the One-Stage Games

\begin{tabular}{lcccc}
\hline \hline Game & Equilibrium & $\begin{array}{c}\text { Average } \\
\text { Individual Payoff }\end{array}$ & $\begin{array}{c}\text { Percentage } \\
\text { Invested }\end{array}$ & Coordination \\
\hline Two-person chicken & $(1,0)^{*}$ & 3.50 & 50.0 & Two players \\
& $(.6, .6)^{*}$ & 2.00 & 60.0 & None \\
Four-person chicken & $(1,0,0,0)^{*}$ & 4.00 & 25.0 & Four players \\
& $(0,0, .2, .2)^{*}$ & 2.70 & 10.0 & Four players \\
& $(0, .11, .11, .11)^{*}$ & 2.55 & 8.3 & Four players \\
Intergroup chicken & $(.072, .072, .072, .072)^{*}$ & 2.50 & 7.2 & None \\
& $(1,0,0,0)^{*}$ & 4.00 & 25.0 & Four players \\
& $(.6, .6 ; 0,0)^{* *}$ & 3.50 & 30.0 & Two teams \\
& $(.42, .42, .42, .42)^{*}$ & 2.70 & 42.0 & None \\
\hline
\end{tabular}

NOTE: See text for explanation of asterisks.

One obvious predictor of the likelihood of cooperation, as defined earlier, is the number of players involved. The prospects of successful coordination decrease as the number of players increases (Hamburger, Guyer, and Fox 1975; Marwell and Schmitt 1972; Oye 1986). Thus, the mere complexity of the (intergroup and single-group) four-person games, as compared to that of the two-person game, renders the realization of common interests in the larger games more difficult.

There is, however, another basis for predicting the prospects of cooperation that is specific to the game of chicken. A basic feature of a chicken game (when some form of communication or signaling among the players is possible) involves the first-mover advantage, that is, the incentive each player has to be the first to bind himself irrevocably to noncooperation. The noncooperative strategy in the two-person chicken game is to invest the endowment; a player who succeeds in making his intention to invest seem convincing is bound to win if the other player is rational (e.g., Rapoport 1997). In the four-person (single-group) chicken game, players who establish themselves as noninvestors can compel the other players to make the investments necessary for provision of the public good (Ward 1990). ${ }^{4}$ In addition to being an effective tactic for the individual player, preemption is an important coordination device for the group as a whole. Introducing asymmetry among the players to match the asymmetry inherent in the pure-strategy equilibrium solutions can help the group avoid the inferior outcome associated with uncoordinated action. Of course, there always is some risk involved; the uncertainty about the behavior of the other player(s) leaves open the possibility of "collision" (in the two-person game) or nonprovision (in the four-person game) in which committed players cannot back down or cannot do so in time (Schelling 1977; Jervis 1978). 
In the intergroup chicken game, where the pure-strategy equilibria are asymmetric both between the two teams and within each team, the situation is considerably more precarious. Each player in this game prefers that his or her team contain the single contributor, but each also prefers that the other team member bear the cost of contribution. Thus, each player has an interest in establishing himself or herself as an investor vis-à-vis the members of the other team and, at the same time, as a noninvestor vis-à-vis the other member of his or her own team. Because these two objectives are contradictory, players in the intergroup chicken game cannot commit themselves credibly to any given action.

To illustrate this point, assume that the members of team $A$ are the first to commit themselves to the competitive "invest" strategy. To the extent that this commitment is perceived as credible by the members of team $B$, they have no incentive to invest their endowments. Here the intergroup conflict has the characteristics of a chicken game as understood by Schelling (1960) and other social psychologists and political scientists. That is, victory can result from a display of the intention to win and the resolve to follow through on that intention. However, the expectation that the members of the opposing team will "chicken out" results in a two-person game of chicken between the members of team $A$, as each prefers to free ride rather than pay the cost of participation. Of course, if the members of team $B$ expect that the members of team $A$ will become involved in this intragroup game, then they might decide to invest their endowments in the hope of winning. Yet the possibility of their implementing this decision depends on their ability to solve their own intragroup chicken game, and so on. In other words, although the players on each team can improve on the Nash equilibrium outcome by coordinating their strategies, no such coordination is selfenforcing (Berenheim, Peleg, and Whinston 1987).

This strategic structure of the intergroup game also is likely to obstruct the effectiveness of reciprocation as a coordination device. Reciprocation, or the tendency to respond to present cooperation (defection) with future cooperation (defection), is effective only to the extent that it is credible (Oye 1986). Credibility, in turn, hinges on the ability of the actors to distinguish reliably between cooperation and defection by others and to respond in kind. Because reciprocity requires flexibility, both control and recognition are important for the successful implementation of tit-for-tat or similar strategies. Unitary players have few control or recognition problems (because the actors can easily distinguish between cooperative and competitive actions of the other players and respond in kind). However, as the preceding scenario illustrates, teams are expected to have difficulties with both.

In sum, our experiment involves two contrasts. First, on the basis of the number of players involved, we expect more cooperation in the two-person game of chicken than in the larger (intergroup and single-group) four-person games. Second, based on the strategic properties of the two four-person games, we predict more cooperation in the single group than in the intergroup chicken game. 


\section{THE EXPERIMENT}

\section{METHOD}

\section{Subjects and Design}

The subjects were 100 male undergraduate students at the Hebrew University of Jerusalem. Subjects were recruited by campus advertisements promising a monetary reward for participation in a decision-making task. Subjects participated in the experiment in groups of four (in the intergroup and four-person game conditions) or two (in the two-person game condition); each condition consisted of 10 groups.

\section{Procedure}

On their arrival at the laboratory, subjects were seated in a single room (in the intergroup condition, they were randomly assigned to the "green" and "red" teams with two subjects on each team). Subjects were given verbal instructions concerning the rules and payoffs of the game (see Tables 1-3). The game instructions were phrased in terms of individual $i$ 's payoffs as a function of his or her own decision to invest or not and the decisions made by the other players in the game. Subjects were not instructed to maximize their earnings, and no reference to cooperation or defection was made. Subjects were given a quiz to test their understanding, and explanations were repeated until the experimenter was convinced that all subjects understood the payoff matrix. Subjects were told in advance that they would receive their payoffs in sealed envelopes and leave the laboratory one at a time with no opportunity to meet the other participants, and they were assured that the experiment involved no deception.

Each group of four or two subjects played 40 rounds (iterations) of the same game. The number of rounds to be played was not known to the participants. Each subject had an electric switch that controlled a green or a red light bulb (according to team membership) on an electric board at the front of the room. At the beginning of each round, all the switches were off. A subject could signal his or her intention to contribute by turning on the electric switch. Subjects had a 30-second period in which they could change their signals as often as they wanted to in real time. When the 30 -second period was up, the board was frozen automatically and subjects were paid according to the decisions made by all the subjects. Following the completion of a round, the decisions were recorded by the experimenter (subjects also recorded the outcome of each round, enabling them to double check their earnings). The lights on the board were turned off and the sequence was repeated.

Following the final round, the points were added up and cashed in at the rate of IS 1 for 5 points ( 1 Israeli shekel equaled approximately U.S. $\$ 0.40$ at the time the experiment took place, and the average earnings per subject were IS 24.34 , about U.S. 


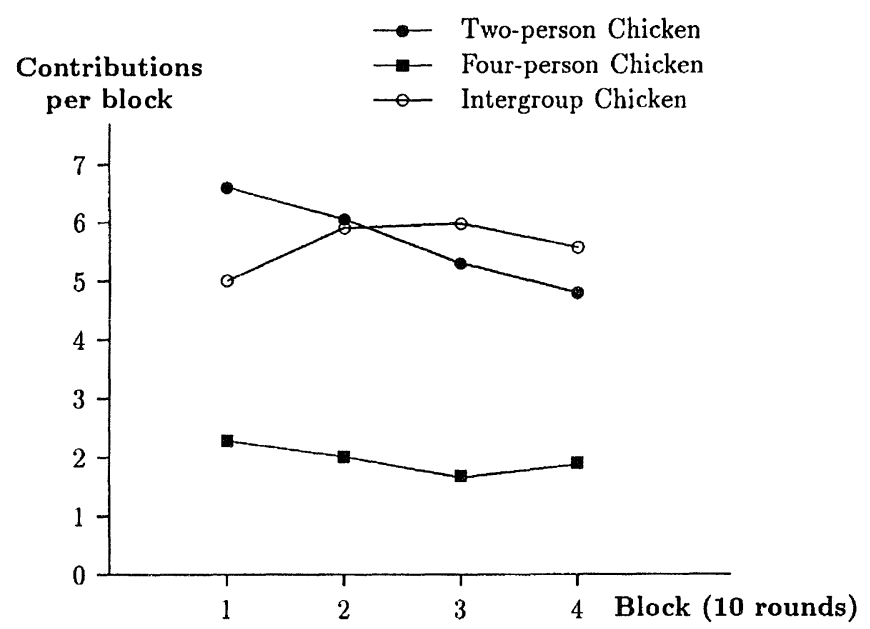

Figure 2: Mean Number of Contributions per Subject per 10-Round Block

\$9.74). Subjects were then briefed on the rationale and purpose of the study and were paid and dismissed individually.

\section{RESULTS}

\section{Contribution Rates and Pareto Efficiency}

Out of the 40 decisions, the mean number of contributions per subject was 22.45 in the intergroup game, 22.75 in the two-person game, and 7.80 in the four-person game. For each game type, the 40 rounds (repetitions) were divided into four blocks of 10 rounds each, and the mean number of contributions per individual subject in each block was calculated. The mean values appear in Figure 2.

The data were analyzed in a two-way analysis of variance (ANOVA) with one between-subjects (type of game) and one within-subjects (block number) factor. This analysis revealed a significant effect of game type, $F(2,97)=51.71, p<.01$, no significant effect for block, $F(3,95)=2.42, p>.01$, and a significant interaction effect, $F(6,190)=4.19, p<.01$. A series of pairwise Tukey's HSD tests indicated that the number of contributions was significantly lower in the four-person condition, but there was no difference between the intergroup and two-person conditions. The finding that subjects in the intergroup game contributed at about the same rate as those in the two-person game should be interpreted in light of the fact that, according to the Pareto-optimal solution, subjects in the intergroup game were predicted to contribute 
TABLE 5

Number of Investors for Each of the Three Games, across Groups and Rounds

\begin{tabular}{cccc}
\hline \hline Number of Investors & Two-Person Chicken & Four-Person Chicken & Intergroup Chicken \\
\hline 0 & $36(9.00)$ & $123(30.75)$ & $5(1.25)$ \\
$\mathbf{1}$ & $\mathbf{2 7 3 ( 6 8 . 2 5 )}$ & $\mathbf{2 4 3}(\mathbf{6 0 . 7 5})$ & $\mathbf{1 0 4}(\mathbf{2 6 . 0 0})$ \\
2 & $91(22.75)$ & $33(8.25)$ & $129(32.25)$ \\
3 & & $1(0.25)$ & $112(28.00)$ \\
4 & & $0(0.00)$ & $50(12.00)$ \\
Total & $400(100.00)$ & $400(100.00)$ & $400(100.00)$ \\
Mean & 1.14 & 0.76 & 2.25 \\
Variance & 0.30 & 0.35 & 2.58 \\
\hline
\end{tabular}

NOTE: Percentages are in parentheses. The Pareto-optimal equilibria are highlighted in bold type.

in $25 \%$ of the rounds, whereas players in the two-person game were predicted to contribute in $50 \%$ of the rounds. The interaction also is highly enlightening in that the number of contributions decreases across blocks for the two-person and four-person games but not for the intergroup game.

Next we turn to an analysis of the results at the group (rather than the individual) level. In each of the three game conditions, 10 sets of subjects played 40 rounds, that is, a total of 400 repetitions for each game. Table 5 summarizes the number of contributors per group as a function of game type.

A very clear pattern emerges. In almost $70 \%$ of the 400 rounds played in the two-person chicken condition, the number of contributors is 1-the Pareto-optimal outcome. In the four-person game, more than $60 \%$ of the rounds resulted in the optimal outcome, and, with a single exception, the deviation from this solution was \pm 1 . The intergroup chicken game, however, induced much higher (and inefficient) rates of contributions. Only $26 \%$ of the rounds resulted in the Pareto-efficient outcome. The modal number of contributors was in fact 2 , and, in more than $40 \%$ of the rounds, the number of contributors was even higher.

The final analysis in this section looks at the actual amounts of money earned by the subjects in the various conditions. Recall that all three games share a common Pareto-optimal solution, namely one contributor. At this equilibrium point, the average payment per subject in the two-person game is 3.5 points (i.e., the contributor wins the game and receives 5 points, whereas the noncontributor keeps his or her 2-point endowment), and the average payment per subject in the four-person and intergroup games is 4 points (i.e., in the four-person game, the single contributor gets 2.5 points, whereas each of the other three players gets 4.5 points; in the intergroup game, the contributor gets 5 points, his or her teammate gets 7 points, and each of the two members of the other team gets 2 points). Table 6 summarizes an individual's average earnings as a function of the game played and the block. To interpret these numbers, keep in mind that the average number of points earned by a member of a group that 
TABLE 6

Mean Number of Points per Player as a Function of Game, Type, and Block

\begin{tabular}{cccc}
\hline \hline Block & Two-Person Chicken & Four-Person Chicken & Intergroup Chicken \\
\hline 1 & 22.80 & 34.95 & 30.25 \\
2 & 24.14 & 32.75 & 26.45 \\
3 & 26.40 & 32.30 & 29.18 \\
4 & 29.40 & 33.75 & 28.85 \\
Total & 102.76 & 133.64 & 119.24 \\
\hline
\end{tabular}

played the equilibrium solution in each and every round is 35 for each block (140 across all blocks) in the two-person game and 40 for each block ( 160 across all blocks) in the four-person and intergroup games. Thus, it is meaningful to compare only the two larger games in terms of the average amount earned per player. Comparing the mean earnings in these two games indeed indicates that subjects earned significantly more in the four-person game than in the intergroup game, $F(1,18)=7.72, p<.01$. It also is interesting to note that the amount earned increases steadily and at an impressive rate only for the two-person game.

\section{Coordination and Turn Taking}

The Pareto-optimal outcome involves a contribution of a single player, whereas a fair and symmetric outcome involves turn taking among all players. Ideally, each player should contribute on every second trial in the two-person game and on every fourth trial in the four-person and intergroup games. How often do individual subjects contribute, and is there a distinct pattern of contributions under the various game conditions? The following analyses attempt to answer these questions. First, we calculated for each subject the lag between two successive contributions. Thus, if a certain subject contributed on trial 1, did not contribute on trials 2-6, and contributed again on trial 7 , then this lag is $(7-1=) 6$. The number of lags varies from person to person because it depends on the subject's total number of contributions. In fact, for those few subjects who never contributed or contributed only once, there are no lags to be analyzed. Table 7 shows the distribution of lags summed over subjects and rounds in each game condition.

The results are quite clear. For the two-person game, the modal lag is 2 (alternate between contributions and noncontributions) with a sizable number of contributions on successive trials $(\mathrm{lag}=1)$. For the four-person game, we have a single peaked distribution at 4 , that is, contributions on every fourth round. In the intergroup chicken game, more than two thirds of the contributions are on adjacent trials. ${ }^{5}$

Next we analyze the evidence for coordination among the members of the various sets. First we report correlations between a subject's own decision to contribute on a

5. The same analysis was repeated after aggregation of within-subjects results. For each individual, we identified the modal lag and tabulated this value as a function of the condition in which he or she participated. These results support the same conclusions. 
TABLE 7

Distribution of Contribution Lag by Type of Game

\begin{tabular}{ccccc}
\hline \hline Lag & Two-Person Chicken & Four-Person Chicken & Intergroup Chicken \\
\hline 1 & 196 & $(45.06)$ & $39(14.18)$ & $583(67.95)$ \\
2 & 210 & $(48.28)$ & $44(16.00)$ & $154(17.95)$ \\
3 & 16 & $(3.68)$ & $51(18.55)$ & $62(7.23)$ \\
4 & 5 & $(1.15)$ & $69(25.09)$ & $23(2.68)$ \\
5 & 3 & $(0.69)$ & $24(8.73)$ & $20(2.33)$ \\
6 & 1 & $(0.23)$ & $12(4.36)$ & 8 \\
7 or more & 4 & $(0.92)$ & $36(13.09)$ & $8.93)$ \\
\hline
\end{tabular}

NOTE: Percentages are in parentheses.

given trial and the decisions of the other players. Because the Pareto-optimal solution requires a single contributor in each set, coordination implies negative correlations between the player's own decision and the decisions of the other players; that is, one should contribute only when this is required (i.e., when the others do not). The aggregate correlations between a player's contribution and the contribution of the other member(s) of the set are $-.39,-.32$, and -.16 for the two-person, four-person, and intergroup chicken games, respectively. When correlations are calculated for each subject separately, the median (individual) values for the three games are -.63, -.34, and -.19, respectively. Thus, in both analyses, the two-person chicken game has the highest level of coordination and the intergroup chicken has the lowest.

Turn taking also means that each player's contribution on a given trial should be negatively correlated with his or her own contributions on previous rounds and positively with other players' contributions on previous rounds. In Table 8, we report these correlations for lags of $k=1,2,3,4$, and 5. The correlations were computed across all players and rounds.

Clearly, the players' contributions in the two-person game correlate positively with their partners' behavior on the previous trial and negatively with their own previous behavior. In other words, subjects within each pair tend to alternate (take turns). To facilitate comparison among the games, we adopted a simple rule for coding behavior of the others in the larger (four-person and intergroup) games: we simply distinguish between cases in which one or more of the other players contributed (without taking into account the exact number of contributors) and those in which none contributed. As can be seen in Table 8, there is some weak evidence for coordination in the four-person game (note that the tendency to contribute is smaller immediately after a contribution and peaks on the fourth trial) but no sequential pattern whatsoever for the intergroup chicken game.

Another implication of coordination is that the number of contributors per round should be constant (namely 1) across trials; in other words, the variance in the number of contributors per round should be low (ideally 0 ). The mean variances were found to be $2.74,3.25$, and 7.81 for the two-person, four-person, and intergroup games, respectively. The data were analyzed by a one-way ANOVA (after a log transformation 
TABLE 8

Correlation Coefficients of Contribution on Trial $i$ with Contribution on Trial $i-k(\mathrm{lag})$ by Type of Game

\begin{tabular}{|c|c|c|c|c|c|c|}
\hline \multirow[b]{2}{*}{$\operatorname{Lag} \mathrm{k}$} & \multicolumn{2}{|c|}{ Two-Person Chicken } & \multicolumn{2}{|c|}{ Four-Person Chicken } & \multicolumn{2}{|c|}{ Intergroup Chicken } \\
\hline & Own & Others & Own & Others & Own & Others \\
\hline 1 & -.29 & .50 & -.08 & .12 & .24 & -.01 \\
\hline 2 & .57 & -.37 & -.02 & .07 & .23 & .01 \\
\hline 3 & -.26 & .41 & .08 & .03 & .23 & 01 \\
\hline 4 & .50 & -.33 & .22 & -.08 & .23 & -.01 \\
\hline 5 & -.22 & .43 & .07 & -.01 & .26 & .00 \\
\hline
\end{tabular}

to stabilize the variances), and we found a significant effect of game, $F(2,26)=8.35$, $p<.01$, with the lowest variance in the two-person chicken and the highest in the intergroup chicken game. ${ }^{6}$

Next we analyzed the number of contributors on a given game as a function of the number of contributors on the previous trial. The analysis was done within the game condition, across all 10 groups and 40 rounds. The trademark of coordination is persistence, that is, repetition of the same strategy and in particular for the Paretooptimal single-contributor solution. The now familiar pattern emerged again: coordination is extremely high ( $77 \%$ overall persistence rate and $60 \%$ persistence rate for the equilibrium) in the two-person chicken game and is lowest for the intergroup chicken game (37\% overall persistence rate and $12 \%$ persistence rate for the equilibrium), with the four-person chicken game in between (52\% overall persistence rate and $39 \%$ persistence rate for the equilibrium). ${ }^{7}$

Our final analysis examines the symmetry in contribution rates among players. We listed for each player his or her proportion of contributions (out of the 40 decisions) and calculated for each set of $K$ players ( $K=2$ or 4, depending on game type) the difference between the highest and lowest contribution proportions. The range for each set and the mean range for each game type appear in Table 9. As can be seen in the table, this range is lowest in the two-person game and highest in the intergroup game.

We also tested the hypothesis that the contribution proportions of the $K$ players in a particular set are equal. The standard test for equality of $K$ proportions is inappropriate in this case because (a) the $K$ proportions are not independent and (b) the 40 rounds are not independent. To overcome the first problem, we used Cochran's (1950) test $(Q)$ of equally matched proportions. To deal with the second problem, we adjusted Cochran's statistic for serial dependence in responses using the procedure described by Budescu (1985). We estimated for each subject the first-order serial

6. In one of the two-person chicken games, we observed perfect coordination; that is, the two players took turns from trials 1 to 40 . This pair has a variance of zero and cannot be used in the ANOVA (because $\log 0$ is undefined). Thus, the results, although significant, underestimate the amount of coordination obtained in this game.

7. Similar results were obtained when the data for the first 20 trials and the last 20 trials were analyzed separately, and so they are not reported here. 
TABLE 9

Differences in Contribution Proportions between the Player with the Highest Proportion and the Player with the Lowest Proportion (ranges of proportions)

\begin{tabular}{lccc}
\hline \hline Set & Two-Person Chicken & Four-Person Chicken & Intergroup Chicken \\
\hline 1 & .5000 & .3250 & .3000 \\
2 & .3000 & .3250 & .4750 \\
3 & .0250 & .3750 & .1250 \\
4 & .0250 & .1000 & .5750 \\
5 & .2500 & .0750 & .2750 \\
6 & .0750 & .1250 & .2000 \\
7 & .0500 & .1750 & .8250 \\
8 & .0250 & .4000 & .3750 \\
9 & .0250 & .0750 & .5500 \\
10 & .0500 & .3500 & .5000 \\
Mean & .1325 & .2275 & .4200 \\
Variance & .0267 & .0169 & .0430 \\
\hline
\end{tabular}

correlation and used the median within-set serial correlation, $C$, to calculate an adjusted statistic, $Q A$, as

$$
Q A=Q(1+C) /(1-C)
$$

The symmetry hypothesis was tested separately for each set. Therefore, to avoid inflated error rates due to multiple tests, we used a more conservative level of significance, namely $\alpha=.05 / 10=.005$. The critical value for rejecting the hypothesis of equality between two proportions is $\chi^{2}(1, .995)=7.88$. In only 1 of the 10 sets in the two-person chicken condition was the hypothesis of symmetry (equality) in contribution proportions rejected; that is, in only one set did the observed $Q A$ value exceed the critical value. In the four-person chicken game, the hypothesis of equal proportions was rejected in 4 of the 10 sets (in testing the equality of four proportions, the critical value for rejection is $\left.\chi^{2}[1, .995]=12.84\right)$.

In the intergroup chicken game, we performed two types of symmetry tests: the symmetry within each dyad (team) and the symmetry among all four players (using the suitable $\chi^{2}$ values). The hypothesis of symmetry within teams was rejected in only 4 of the 20 teams. On the other hand, the hypothesis of symmetry among the four participants was rejected in 6 of 10 sets.

\section{DISCUSSION}

We contrasted the intergroup chicken game with two control conditions: a twoperson chicken and an $n$-person (single-group) chicken game. As predicted, the level of cooperation in the intergroup game was lower than that in either the two-person or the $n$-person game. We discuss these two contrasts in turn. In the two-person game, 
almost $70 \%$ of the rounds resulted in the Pareto-optimal outcome of one player contributing, and in many of the pairs this proportion was much higher. There also was a clear indication of learning as the level of efficiency and, consequently, the amounts of money earned increased steadily as the game progressed. Finally, turn taking between the two players was the rule rather than the exception. These results stand in sharp contrast to those observed in the intergroup competition, as only $26 \%$ of the rounds resulted in the Pareto-optimal outcome; practically all of the other rounds resulted in a higher, and therefore inefficient, rate of contribution; and, most notably, $12.5 \%$ of the rounds resulted in a full-scale "collision" of all four players contributing. In addition, there was little indication of turn taking among the participants within teams or between teams, and there were no signs of improvement over time.

It can be argued that the differences between the two-person and intergroup chicken games are due to the fact that the intergroup game involves a larger number of players and therefore entails a more intricate coordination problem. However, the results in our second control condition attest against this possibility. The contrast involving the intergroup and four-person chicken games is a straightforward one. Both games involve the same solution and the same number of players and therefore present subjects with an identical coordination problem: to achieve the Pareto-optimal outcome, a single player should contribute on each round of the game; to obtain fair outcomes, the players should alternate in taking this role. Yet, as our results clearly indicate, there was better coordination in the single-group game than in the intergroup game. More than $60 \%$ of the rounds in the former game resulted in the Pareto-optimal outcome as compared to only $26 \%$ in the latter. Obviously, players did not treat these two games as an identical coordination problem.

Can the results be explained by the notion of mixed strategy? As indicated in the introductory paragraphs of this article, according to the symmetric Nash equilibrium in mixed strategies, subjects should contribute with probabilities of $p$ 's $=.42, .60$, and .07 in the intergroup, two-person, and four-person chicken games, respectively. If each subject indeed contributes according to these probabilities independently of the other $n-1$ players, then the total number of contributors is expected to be a binomial with $n$ being the number of players and $p$ being the probability of contribution according to the mixed-strategy equilibrium for each game. Thus, for example, we would expect the number of contributors in the two-person chicken game to follow a binomial distribution with $n=2$ and $p=.60$; that is, $16 \%$ of the games should have no contributors, $48 \%$ of the cases should have exactly one contributor, and the remaining $36 \%$ should have both players contributing. Similarly, the number of contributors in the intergroup chicken game is given by a binomial distribution with $n=4$ and $p=$ .42 ; in the four-person game, it is given by a binomial with $n=4$ and $p=.07$. To test the hypothesis that subjects followed this strategy, we compared the expected (binomial) distribution to the actual number of contributors per game (across groups and trials). The null hypothesis was soundly rejected (at the .01 level) by a KolmogorovSmirnov test in all cases ( $D$ 's $=.13, .44$, and .20 for the two-person, four-person, and intergroup chicken games, respectively). Thus, the results cannot be explained by use of mixed strategies alone. 
An interesting result is that failure to coordinate on the Pareto-optimal solution was likely to result in overprovision in the two-person and intergroup games but in underprovision in the four-person game. Specifically, of the 127 nonoptimal rounds in the two-person game, $91(72 \%)$ resulted in both players contributing, whereas 36 $(28 \%)$ of the rounds resulted in neither player contributing. In the intergroup game, there were 296 nonoptimal rounds, and 291 (almost 99\%) of them resulted in overprovision (i.e., two or more players contributing). By contrast, of the 157 nonoptimal rounds in the single-group four-person game, $123(78 \%)$ resulted in underprovision (i.e., no contributors).

We can offer two explanations for these results. The first is based on the dynamic nature of the repeated game. A repeated game of chicken entails long-term considerations of reputation; each player may refrain from yielding in the present to force the other player(s) to yield in the future. Establishing a reputation of toughness may be costly, but if the "shadow of the future" (Axelrod 1984) is long enough, it might be well worth the cost. As explained earlier, reputation considerations may lead a player to contribute (even if other player also does) in the two-person game and to withhold contribution (even if no one else contributes) in the four-person game. The reputation effect is thus consistent with overprovision in the former game and underprovision in the latter.

In the intergroup chicken game, establishing a reputation of toughness is more problematic. Each player has an interest to appear as an investor vis-à-vis the members of the other team and as a noninvestor vis-à-vis the other members of his own team. The overprovision observed in this condition suggests that establishing reputation of toughness vis-à-vis the opposing team may be the more important consideration. This interpretation receives support from studies involving between-dyad play in a mixedmotive game followed by within-dyad play of the same game for division of winnings (Doise 1978; Wilson and Kayatani 1968). These studies found consistently more competition between the two-person groups than within the groups.

The dynamics observed in one of our experimental sessions illustrates this point quite nicely. In this particular session, one of the teams (team $A$ ) established its dominance quite early in the game. After a few collisions with the opposing team (team $B)$, team $A$ began to win one round after another. The scenario became quite predictable (perhaps even boring); at the beginning of each 30 -second signaling period, all four lights went on, indicating an intention by all players to contribute, but before the time was up one of the players in team $B$ turned off his or her light and the other immediately followed. The two members of team $A$, however, did not use this opportunity to free ride. Rather than get involved in the internal game of chicken, both kept their lights on until the end of the period. Although this strategy can be considered wasteful (because both players in team $A$ lost their endowments), it was quite effective in deterring the other team.

Another explanation is based on the symmetric mixed-strategy equilibrium of the static and repeated game. It is possible that when coordination on the pure-strategy equilibrium fails, players resort to the use of mixed strategies. Although we were able to reject the null hypothesis that mixed strategies can explain the behavior observed 
throughout the entire game, it is nonetheless possible that, when coordination failed, mixed strategies exerted some pull on the subjects' behavior. The mixed-strategy equilibrium predicts contribution with low probability (.07) in the four-person game and with relatively high probability in the two-person and intergroup games (.60 and .42 , respectively). These predictions are consistent with the observed pattern of results. Recall that if subjects contributed independently with these probabilities, then the expected number of contributions in the four-person game is .28 (4 players $\times .07$ ), which is less than 1 (i.e., underprovision). On the other hand, the expected number of contributions in the two-person and intergroup chicken games is 1.2 (2 players $\times .60)$ and 1.68 (4 players $\times .42$ ), respectively. Both values are greater than 1 and so predict overprovision. It is, of course, possible for both factors to operate simultaneously, but it is impossible to distinguish the relative contributions of the two effects based on this study.

\section{CONCLUSION}

The problem of collective action in intergroup conflict has been largely ignored by the existing literature. The literature on social dilemmas and public goods provision traditionally has treated these problems as single-group $n$-person games. Even researchers who acknowledge that one group's public good often is another's public "bad" (Hardin 1982; Fireman and Gamson 1979) do not integrate this additional dimension of intergroup conflict into their models of individual and group decision making. On the other hand, strategic analyses that represent intergroup conflict as two-person games overlook the collective action problem within the competing groups. Despite the recognition that the unitary player assumption is too simplistic, most researchers are reluctant to drop this assumption because of the fear that this would result in models that are too complex for heuristic or explanatory purposes (Bendor and Hammond 1992).

The team game approach overcomes the limitations of the standard paradigms. It integrates the intragroup and intergroup levels of analysis and enables researchers to investigate systematically the interaction between internal organization and outside conflict. The results of the present study accentuate the importance of this integrated framework. Clearly, the intergroup chicken game is not played out as a two-person game of chicken between unitary players, nor is it treated as a four-person game of chicken among four individuals. Rather, the conflict of interests within the groups intensifies the conflict between the groups, and the conflict between the groups increases the competition among the individual players. Similar results were obtained in a recent study by Bornstein and Ben-Yossef (1994) that compared the intergroup prisoner's dilemma group game with a single-group prisoner's dilemma game. If nothing else, these team game experiments suggest that extrapolation from experiments with two-person and $n$-person games to intergroup conflicts could be misleading because it provides a prediction that is far too optimistic about the prospects of cooperation. 


\section{APPENDIX \\ Nash Equilibrium Points}

In this Appendix, we find all Nash equilibrium points of each of the three variants of chicken game studied in this article.

\section{TWO-PERSON CHICKEN GAME}

Given that player 1 invests with probability $p$, the expected payoff for player 2 is $0 \bullet p+5(1-$ $p$ ) if he or she invests and 2 if he or she does not invest. Hence, player 2's best reply is to invest if $5(1-p)>2$ (i.e., $p<.60$ ). In other words, if we denote by $b(p)$ the probability of investing in the best reply to $p$, then the best reply correspondence $b(p)$ is given by

$$
b(p)= \begin{cases}1 & \text { if } p<0.6 \\ {[0,1]} & \text { if } p=0.6 \\ 0 & \text { if } p>0.6\end{cases}
$$

A Nash equilibrium is a pair of probabilities $(p, q)$ such that $q \in b(p)$ and $p \in b(q)$. It is easily seen that the only three equilibria are $(1,0),(0,1)$, and $(.6, .6)$.

\section{FOUR-PERSON CHICKEN GAME}

Denote by $p_{i}$ the probability of player $i$ to invest and the general strategy profile by $p=\left(p_{1}, p_{2}\right.$, $\left.p_{3}, p_{4}\right)$.

Proposition 5.1: The set of all Nash equilibria of the four-person chicken game consists of the following:

(i) $p=(1,0,0,0)$ and all its permutations (pure-strategy equilibria).

(ii) $p=\left(p^{*}, p^{*}, p^{*}, p^{*}\right)$, where $p^{*}=1-\sqrt[3]{0.8}=0.072$ (symmetric mixed-strategy equilibrium).

(iii) $p=\left(0, q^{*}, q^{*}, q^{*}\right)$ and all its permutations, where $q^{*}=1-\sqrt{0.8}=.11$.

(iv) $p=(0,0, .2, .2)$ and all its symmetries.

Proof. If players 2,3 , and 4 invest with probabilities $p_{2}, p_{3}$, and $p_{4}$, respectively, and if player 1 does not invest, then his or her expected payoff is

$$
2\left(1-p_{2}\right)\left(1-p_{3}\right)\left(1-p_{4}\right)+4.5\left[1-\left(1-p_{2}\right)\left(1-p_{3}\right)\left(1-p_{4}\right)\right] .
$$

If player 1 does invest, then his or her payoff is 2.5 . Therefore, player 1's best reply correspondence is

$$
b\left(p_{2}, p_{3}, p_{4}\right)= \begin{cases}0 & \text { if }\left(1-p_{2}\right)\left(1-p_{3}\right)\left(1-p_{4}\right)<0.8 \\ {[0,1]} & \text { if }\left(1-p_{2}\right)\left(1-p_{3}\right)\left(1-p_{4}\right)=0.8 \\ 1 & \text { if }\left(1-p_{2}\right)\left(1-p_{3}\right)\left(1-p_{4}\right)>0.8\end{cases}
$$

and similarly for the other players. 
If for any $i \in\{1,2,3,4\}$ and $\{p 1, p 2, p 3, p 4\}$ we let $p_{-i}=\left\{p_{1}, p_{2}, p_{3}, p_{4}\right\} \backslash\left\{p_{i}\right\}$, then $p=$ $\left(p_{1}, p_{2}, p_{3}, p_{4}\right)$ is a Nash equilibrium if and only if $p_{i} \in b\left(p_{-i}\right)$ for $i=1,2,3,4$.

Let $p=\left(p_{1}, p_{2}, p_{3}, p_{4}\right)$ be a Nash equilibrium.

(i) If $p_{1}=1$, then (by the symmetrics of condition 1) $p_{2}=p_{3}=p_{4}=0$, which yields the pure-strategy equilibrium $(1,0,0,0)$ and all its symmetries. The remaining equilibria must therefore satisfy $p_{i}<1 \forall i$.

(ii) If $0<p_{1}<1$ for all $i$, then (by condition 1 )

$$
0<p_{1}<1 \text { implies }\left(1-p_{2}\right)\left(1-p_{3}\right)\left(1-p_{4}\right)=.8 \text {. }
$$

Applying this for all players, we must have the following:

$$
\begin{gathered}
\left(1-p_{1}\right)\left(1-p_{2}\right)\left(1-p_{3}\right)=.8, \\
\left(1-p_{4}\right)\left(1-p_{2}\right)\left(1-p_{3}\right)=.8, \\
\left(1-p_{4}\right)\left(1-p_{2}\right)\left(1-p_{1}\right)=.8, \text { and } \\
\left(1-p_{4}\right)\left(1-p_{1}\right)\left(1-p_{3}\right)=.8,
\end{gathered}
$$

which imply $p_{1}=p_{2}=p_{3}=p_{4}=p^{*}$, where $p^{*}$ satisfies $\left(1-p^{*}\right)^{3}=.8$, that is, $p^{*}=1-$ $\sqrt[3]{0.8}=.072$. It follows that this is not only the unique symmetric Nash equilibrium but also the unique Nash equilibrium in which all players use mixed strategies. The remaining equilibria must thus satisfy $p_{i}<1$ for all $i$ and $p_{i}=0$ for at least one $i$.

(iii) Assume that only one component is zero, say $p_{1}=0$, and $0<p_{i}<1$ for $i=2,3,4$. Then (by condition 1)

$$
\begin{gathered}
\left(1-p_{3}\right)\left(1-p_{4}\right)=.8, \\
\left(1-p_{3}\right)\left(1-p_{2}\right)=.8, \text { and } \\
\left(1-p_{2}\right)\left(1-p_{4}\right)=.8,
\end{gathered}
$$

which imply $p_{2}=p_{3}=p_{4}=q^{*}$, where $q^{*}$ satisfy $\left(1-q^{*}\right)^{2}=.8$, that is, $q^{*}=1-\sqrt{0.8}=.11$, and $p=\left(0, q^{*}, q^{*}, q^{*}\right)$ is in fact a Nash equilibrium because condition 1 also is satisfied for player 1 . Because $\left(1-q^{*}\right)\left(1-q^{*}\right)\left(1-q^{*}\right)=.8\left(1-q^{*}\right)<.8$, player 1's best reply is $p_{1}=0$.

(iv) If two components of $p$ are zero, say $p_{1}=p_{2}=0$, and $0<p_{i}<1$ for $i=3,4$, then by condition $1\left(1-p_{3}\right)=.8$ and $\left(1-p_{4}\right)=.8$, that is, $p_{3}=p_{4}=.2$, and $(0,0, .2, .2)$ is in fact a Nash equilibrium because condition 1 for players 1 and 2 also is satisfied; that is, because $\left(1-p_{3}\right)\left(1-p_{4}\right)=.8^{2}<.08$, player 1 's and player 2 's best replies are $p_{1}=0$ and $p_{2}=0$, respectively.

Finally, if three components of $p$ are zero, then the fourth player's best reply is 1 and hence this is the pure-strategy equilibrium in condition 1 . We conclude that all the equilibria of the game are those in items (i), (ii), (iii), and (iv) of the proposition. 


\section{INTERGROUP CHICKEN}

Assume that the teams are $\{1,2\}$ and $\{3,4\}$. Denote by $p=\left(p_{1}, p_{2}, q_{1}, q_{2}\right)$ the probabilities of the players to invest. Given $\left(p_{2}, q_{1}, q_{2}\right)$, the expected payoff of player 1 is $5\left[\left(1-q_{1}\right)\left(1-q_{2}\right)+\right.$ $\left.p_{2} q_{1}\left(1-q_{2}\right)+p_{2} q_{2}\left(1-q_{1}\right)\right]$ if he or she invests and $2+5 p_{2}\left(1-q_{1}\right)\left(1-q_{2}\right)$ if he or she does not invest. Therefore, player 1 invests or not depending on the direction of the inequality

$$
5\left[\left(1-q_{1}\right)\left(1-q_{2}\right)+p_{2} q_{1}\left(1-q_{2}\right)+p_{2} q_{2}\left(1-q_{1}\right)\right] \stackrel{\geq}{<} 2+5 p_{2}\left(1-q_{1}\right)\left(1-q_{2}\right),
$$

which is equivalent to

$$
\left(1-p_{2}\right)\left(1-q_{1}\right)\left(1-q_{2}\right)+p_{2} q_{1}\left(1-q_{2}\right)+p_{2} q_{2}\left(1-q_{1}\right) \geq 0.4
$$

Thus, the best reply correspondence of player 1 is

$b\left(p_{2} ; q_{1}, q_{2}\right)= \begin{cases}0 & \text { if }\left(1-p_{2}\right)\left(1-q_{1}\right)\left(1-q_{2}\right)+p_{2} q_{1}\left(1-q_{2}\right)+p_{2} q_{2}\left(1-q_{1}\right)<0.4 \\ {[0,1]} & \text { if }\left(1-p_{2}\right)\left(1-q_{1}\right)\left(1-q_{2}\right)+p_{2} q_{1}\left(1-q_{2}\right)+p_{2} q_{2}\left(1-q_{1}\right)=0.4 \\ 0 & \text { if }\left(1-p_{2}\right)\left(1-q_{1}\right)\left(1-q_{2}\right)+p_{2} q_{1}\left(1-q_{2}\right)+p_{2} q_{2}\left(1-q_{1}\right)>0.4\end{cases}$

and with the apropriate permutations for other players.

We shall find all Nash equilibria and arrange them according to the number of players who do not invest, that is, the number of zero coordinates in $p$. Clearly, $(0,0 ; 0,0)$ is not an equilibrium because (by condition 2) $b(0 ; 0,0)=1$.

(i) Three players do not invest and the fourth player does invest. Because $b(0 ; 0,0)=1$, this yields the pure-strategy equilibrium $(1,0 ; 0,0)$ and all its permutations.

(ii) Equilibrium in which exactly two players do not invest. This splits into two subcases:

(a) Two members of the same team, say team $\{3,4\}$, do not invest. By condition 2, we get $(1,0 ; 0,0)$ or $(0,1 ; 0,0)$, which are already in item (i), and $(.6, .6 ; 0,0)$. This has a very clear interpretation: if it is known that the members of the other team are not investing, then the game between players 1 and 2 is equivalent to the two-person chicken game with the equilibria $(1,0),(0,1)$, and $(.6, .6)$. To complete the argument that $(.6, .6 ; 0,0)$ is a Nash equilibrium, we have to show that $0 \varepsilon b(0 ; .6, .6)$, which is in fact true because $(1-.6)(1-.6)<.4$.

(b) Two players of different teams, say players 2 and 4, are not investing. Here again, the game becomes a two-person chicken game between the other two players with the pure-strategy equilibria being $(1,0 ; 0,0)$ and $(0,0 ; 1,0)$ in item (i). Note, however, that the third candidate for an equilibrium, namely $(.6,0 ; .6,0)$, is not an equilibrium because $b(.6 ; .6,0)=1$ as follows from condition 2 given that $(1-.6)(1-.6)+$ $(.6)(.6)=.52>.4$.

(iii) Only one player does not invest, say $\mathrm{p}_{I}=0$. If $\left(p_{1}, 0 ; q_{1}, q_{2}\right)$ is a Nash equilibrium, then it must be that $q_{1}<1$ and $q_{2}<1$ because (by condition 2$) b\left(0 ; 1, q_{2}\right)=0$. Thus, again by condition 2 ,

$$
\left(1-q_{1}\right)\left(1-p_{1}\right)+q_{1} p_{1}=.4 \text { and }
$$




$$
\left(1-q_{2}\right)\left(1-p_{1}\right)+q_{2} p_{1}=.4,
$$

which implies $\left(q_{1}-q_{2}\right)\left(1-2 p_{1}\right)=0$, which in turn implies that either $p_{1}=.5$ or $q_{1}=q_{2}$. (a) $p_{1}=.5$ is impossible because it contradicts condition $4:\left(1-q_{1}\right) / 2+q_{1} / 2=.4$ implies $.5=.4$.

(b) $q_{1}=q_{2}=q$. Note that $0<p_{1}<1$ (because $p_{1}=1$ and $p_{2}=0$ would imply $q_{1}=q_{2}=.4$, which in turn would imply, by condition $2, p_{1}=0$ ). It follows (by condition 2 ) that $(1-q)^{2}=.4$ and hence $q=1-\sqrt{0.4}=.37$ and (again by condition 2$)(1-.37)(1-$ $\left.p_{1}\right)+.37 p_{1}=.4$ and consequently $p_{1}=.88$. So the only candidate for equilibrium in this class is $(.88,0 ; .37, .37)$. For this to be an equilibrium, $p_{2}=0$ must be a best reply to $(.88 ; .37, .37)$. But

$$
(1-.88)(1-.37)+2(.88)(1-.37)=1.15>.4 \text {. }
$$

Hence the best reply for $(.88 ; .37, .37)$ is $p_{2}=1$. It follows that there is no equilibrium with precisely one player not investing.

(iv) Equilibria in which all players invest with positive probability, namely $p=\left(p_{1}, p_{2}, q_{1}\right.$, $q_{2}$ ), where all components are strictly positive. A careful check shows that no component can be 1 (otherwise condition 2 would be violated). The following follow from condition 2:

$$
\begin{aligned}
& \left(1-p_{2}\right)\left(1-q_{1}\right)\left(1-q_{2}\right)+p_{2} q_{2}\left(1-q_{2}\right)+p_{2} q_{2}\left(1-q_{1}\right)=.4, \\
& \left(1-p_{1}\right)\left(1-q_{1}\right)\left(1-q_{2}\right)+p_{1} q_{1}\left(1-q_{2}\right)+p_{1} q_{2}\left(1-q_{1}\right)=.4, \\
& \left(1-q_{1}\right)\left(1-p_{1}\right)\left(1-p_{2}\right)+q_{1} p_{2}\left(1-p_{2}\right)+q_{1} p_{2}\left(1-p_{1}\right)=.4, \text { and } \\
& \left(1-q_{2}\right)\left(1-p_{1}\right)\left(1-p_{2}\right)+q_{2} p_{1}\left(1-p_{2}\right)+q_{2} p_{2}\left(1-p_{1}\right)=.4 .
\end{aligned}
$$

From conditions 7 and 8 , we get $\left(p_{1}-p_{2}\right)\left(1-q_{1}\right)\left(1-q_{2}\right)=.4\left(p_{1}-p_{2}\right)$, so that either $\left(1-q_{1}\right)\left(1-q_{2}\right)=.4$ or $p_{1}=p_{2}$. The first possibility, $\left(1-q_{1}\right)\left(1-q_{2}\right)=.4$, leads to a contradiction of conditions 7 and 8 , hence $p_{1}=p_{2}=p$ and similarly $q_{1}=q_{2}=q$. It follows that

$$
\begin{gathered}
(1-p)(1-q)+2 p q(1-q)=.4 \\
(1-q)(1-p)+2 p q(1-p)=.4
\end{gathered}
$$

and consequently that $(1-p)=(1-q)$, that is, $p=q$. Hence, the only Nash equilibrium in which all players invest with positive probability is the completely mixed symmetric equilibrium: $p_{1}=p_{2}=q_{1}=q_{2}=p^{*}$ is a solution of $(1-p)^{3}+2 p(1-p)^{2}=.4$, which has only one real solution: $p^{*}=.42$.

We conclude that all Nash equilibria of the game are as follows:

(i) pure strategy equilibrium $p=(1,0 ; 0,0)$ and all its symmetries,

(ii) $(.6, .6 ; 0,0)$ and $(0,0 ; .6, .6)$, and

(iii) the completely symmetric mixed equilibrium $p=(.42, .42 ; .42, .42)$. 


\section{REFERENCES}

Allison, G. 1971. Essence of decision: Explaining the Cuban missile crisis. Boston: Little, Brown.

Axelrod, R. 1984. The evolution of cooperation. New York: Basic Books.

Bendor, J., and T. F. Hammond. 1992. Rethinking Allison's models. American Political Science Review 86:301-22.

Berenheim, B. D., B. Peleg, and M. Whinston. 1987. Coalition-proof Nash equilibria. Journal of Economic Theory 42:1-12.

Bornstein, G., and M. Ben-Yossef. 1994. Cooperation in intergroup and single group social dilemmas. Journal of Experimental Social Psychology 30:52-67.

Brams, S. 1975. Game theory and politics: International relations games. New York: Free Press.

Budescu, D. V. 1985. Analysis of dichotomous variables in the presence of serial dependence. Psychological Bulletin 97:547-61.

Cochran, W. G. 1950. The comparison of percentages in matched samples. Biometrika 37:256-66.

Dieckman, A. 1985. Volunteer's dilemma. Journal of Conflict Resolution 29:605-10.

Doise, W. 1978. Groups and individuals: Explanations in social psychology. Cambridge, England: Cambridge University Press.

Fireman, B., and W. Gamson. 1979. Utilitarian logic in the resource mobilization perspective. In The Dynamics of Social Movements, edited by M. Zald and J. McCarthy. Cambridge, England: Winthrop.

Hamburger, H., M. S. Guyer, and J. Fox. 1975. Group size and cooperation. Journal of Conflict Resolution 19:503-31.

Hardin, G. 1982. The limits of altruism: An ecologist's view of survival. Bloomington: Indiana University Press.

Jervis, R. 1978. Cooperation under the security dilemma. World Politics 30:167-86.

Kahneman, D., J. L. Knetsch, and R. Thaler. 1986. Fairness and the assumptions of economics. Journal of Business 59:285-300.

Marwell, G. S., and D. Schmitt. 1972. Cooperation in a three-person prisoner's dilemma. Journal of Personality and Social Psychology 21:376-83.

Oye, K. A. 1986. Explaining cooperation under anarchy: Hypotheses and strategies. In Cooperation under Anarchy, edited by K. A. Oye. Princeton, NJ: Princeton University Press.

Palfrey, T., and H. Rosenthal. 1983. A strategic calculus of voting. Public Choice 41:7-53.

1991. Testing for effects of cheap talk in a public goods game with private information. Games and Economic Behavior 3:183-220.

Rasmusen, E. 1989. Games and information: An introduction to game theory. Oxford, England: Basil Blackwell.

Rapoport, A. 1997. Order of play in strategically equivalent game in extensive form. International Journal of Game Theory 26:113-136.

Rubinstein, A. 1980. Strong perfect equilibrium in supergames. International Journal of Game Theory 9:1-12.

Schelling, T. 1960. The strategy of conflict. Cambridge, MA: Harvard University Press. 1977. Micromotives and macrobehavior. New York: Norton.

Snidal, D. 1991. Relative gains and the pattern of international cooperation. American Political Science Review 85:701-26.

Taylor, M. 1987. The possibility of cooperation. Cambridge, England: Cambridge University Press.

van de Kragt, A.J.C., J. M. Orbell, and R. M. Dawes. 1983. The minimal contributing set as a solution to public goods problems. American Political Science Review 77:112-22.

Ward, H. 1990. Three men in a boat, two must row: An analysis of three-person chicken pre-game. Journal of Conflict Resolution 34:371-400.

Wilson, W., and M. Kayatani. 1968. Intergroup attitudes and strategies in games between opponents of the same or of a different race. Journal of Personality and Social Psychology 9:24-30. 